\title{
Type B intramural hematoma: focus on reasons for development and overlapping clinical disease
}

\author{
Martin Haensig \\ Department of Vascular Surgery, University of Leipzig, Leipzig, Germany \\ Correspondence to: Martin Haensig, MD. Department of Vascular Surgery, Cardiovascular Center, University of Leipzig, Liebigstr. 20, 04103 Leipzig, \\ Germany. Email: mhaensig@gmail.com.
}

Submitted Mar 27, 2019. Accepted for publication May 31, 2019.

doi: 10.21037/acs.2019.06.04

View this article at: http://dx.doi.org/10.21037/acs.2019.06.04

Acute aortic syndrome includes a variety of overlapping pathologies. Aortic intramural hematoma (IMH) is described by hemorrhage within the medial layer of the aortic wall, without evidence of flow or clear luminal communication at imaging or surgery (1). The main symptom in more than $90 \%$ of the patients is abrupt or sudden excruciating anterior or posterior chest pain that may radiate to the neck, back, or chest, characterized as aortic pain (2,3). Although IMH accounts for $4 \%$ to $11 \%$ of acute aortic syndromes in the United States and Europe, the occurrence is as high as $32 \%$ in Asia (4). Type A IMHs involve the ascending aorta or the aortic arch $(30 \%$ and $10 \%$ of cases, respectively), whereas on the other side, type $\mathrm{B}$ hematomas are restricted distal to the left subclavian artery, in approximately $60 \%$ of cases $(5,6)$. This review focuses on type B intramural hematomas, with a spotlight on reasons for the development and overlapping of clinical and anatomical diseases.

While normal aortic wall thickness is less than $3 \mathrm{~mm}$, IMH is characterized by a smooth, bow-shaped thickening of the aortic wall diameter of more than $5 \mathrm{~mm}$. The extent of the hemorrhage within the aortic wall can range from one centimeter to the full length of the aorta. Although trauma is occasionally implicated, the vast majority (90\%) of IMH cases are spontaneous (i.e., nontraumatic). Aortic IMH was first described in Leipzig, Germany by Kruckenberg in 1919 as "dissection without intimal tear" that results from hemorrhage within the aortic wall (7). It has been suggested that an IMH results from the spontaneous rupture of vasa-vasorum in the medial layer due to a combination of wall stress, fragile vessels, chronic hypertension and inflammation. More recently, perhaps due to improved imaging technology and resolution, small intimal tears have been detected in as many as $70 \%$ to $80 \%$ of IMH cases (8) and may represent a site of initial insult. As a consequence, some consider an IMH to be a subset of aortic dissection (AD) with little or no flow in the false lumen. These intimal defects have been referred to as ulcer-like projections (ULP) or focal intimal disruptions (FID) and can be found at initial presentation or during follow-up.

ULPs have a large neck $(>3 \mathrm{~mm})$ and protrude from the lumen into the thrombosed media, potentially leading to adventitial bulging and even resulting in a pseudo-aneurysm formation or rupture. Another type of intimal defect seen in some cases of IMH is termed intramural blood pool (IBP), which probably represent pseudoaneurysms of intercostal or lumbar arteries (9). Distinguishing an IMH from other differential diagnosis is important in the determination of prognosis and management. A classic $\mathrm{AD}$ has an intimomedial flap separating a true and false lumen that are in communication with one or more defects, with blood flow presenting in the majority of the time in the false lumen. Intimal calcification is typically displaced as a characteristic of $\mathrm{AD}$ and $\mathrm{IMH}$. Therefore, central displacement of intimal calcification can help to distinguish IMH from mural atheroma/thrombus.

PAU is the result of ulceration through an atheromatous plaque with or without focal outward bulging of the adventitia with a surrounding inflammatory surrogate (10). Approximately $5 \%$ of spontaneous IMH cases are associated with erosion of a PAU into the media with subsequent hemorrhage. Although an IMH may occur throughout the aorta, a PAU is rarely found in the ascending aorta and is infrequently seen in the arch, whereas $\geq 90 \%$ are seen in the 
descending aorta. Moreover, aortitis with mural aortic wall enhancement typically seen at MRI and CT combined with the clinical and laboratory findings suffice for determining the appropriate diagnosis. Sometimes it can be classified as idiopathic, but in cases of infectious aortitis, can show rapid progress with a poor short-term prognosis. Rarer differential diagnoses should be considered, as they require specific care: Takayasu arteritis, giant cell arteritis, Bechet's disease or retroperitoneal fibrosis (mostly idiopathic, but also secondary to neoplasia or malignant hemopathies).

Uchida et al. found that the layer of medial separation in IMHs is nearer to the adventitia than those of classic $\mathrm{AD}$ (11). Due to the pathophysiologic mechanism of IMHs involving a weakened outer medial layer near the adventitia, there is a high risk for developing aneurysms or rupture. Thus, in $28-47 \%$ of cases, IMH progresses to acute $\mathrm{AD}$ (12). Furthermore, IMH is also often associated with peri-aortic hematomas, where blood is found outside the aortic adventitia. The presence of peri-aortic hematoma and pleural effusion can make it difficult to differentiate between the outer wall and rupture of the aortic adventitia. While the presence of a peri-aortic hematoma is a poor prognostic sign and has been associated with the risk of rupture, the presence of pleural effusion may represent reactive fluid collection and is not associated with poor outcomes unless it increases in size.

Although the majority of IMH cases are symptomatic, a few are asymptomatic and incidentally diagnosed at imaging conducted for an unrelated reason (1). Contrast-enhanced CT is valuable in confirmation of an intimomedial flap in $\mathrm{AD}$, for distinguishing $\mathrm{IMH}$ from PAU, or for visualizing ULPs and IBPs, respectively. A heterogeneous or echolucent appearance during ultrasound or CT imaging might be indicative of an unstable IMH (13). To differentiate IMH from aortitis, pre- and post-contrast $\mathrm{CT}$ images are valuable, as IMH will have high attenuation on both $(>50 \mathrm{HU})$, whereas aortitis will have low attenuation on pre-contrast images $(<40 \mathrm{HU})$ with post-contrast enhancement. On T1-weighted MRI images, aortitis shows hypointensity, whereas IMH shows increased signal intensity (14). The benefit of an MRI is limited in the acute setting, however, MRI remains an excellent choice to follow PAUs or IMHs over the long-term (12). Small IMHs may be distinguished by MRI, whereas they could potentially be overlooked on CTA. Early in the formation of IMH, the hematoma shows an iso-intensity on T1 imaging and hyper-intensity on T2 imaging. After the first 1-2 days, however, oxyhemoglobin is converted to methemoglobin leading to a hyper-intense signal in both T1 and T2 MRI images, making MRI an excellent tool for assessing the age of the IMH. This stands in contrast to a mural thrombus, which presents hypo-intense or iso-intense in both T1 and T2 images. Specific MRI techniques may be helpful in differentiating an IMH from atherosclerotic wall thickening, thrombosed dissection and mural thrombus when CT proves to be unsuitable (15).

With an increased detection of intimal tears due to improved imaging resolution, consideration of thoracic endovascular aortic/aneurysmal repair (TEVAR) for uncomplicated type B IMH has increased in the same manner as it has for type B AD. Medical therapy and a close radiographic and clinical follow-up for type B IMH are preferred unless complications are present or foreseen. Nearly $90 \%$ of type B IMH patients are managed medically (best medical treatment; BMT) and less than $10 \%$ surgically (4). The new presence of ULPs, especially with a communicating orifice more than $3 \mathrm{~mm}$ in size, need serial imaging and close follow-up as they are an indicator of disease progression and may benefit from early TEVAR or open repair (16).

For high-risk type B IMH patients, defined as a maximum aortic diameter $>40-45 \mathrm{~mm}$, PAU, hematoma of $>10 \mathrm{~mm}$ at the initial presentation, progressive hematoma in 2 successive imaging studies, uncontrollable hypertension and therapy refractory pain, a more aggressive treatment strategy is recommended. Considering that there are still no RCTs, combined data from current studies demonstrate that TEVAR + BMT results in significantly improved regression rate and lower IMH progression, as well as a lower aortic-related death rate compared with BMT alone in high-risk type B IMH (17). In the chronic phase, an aortic diameter of more than $50 \mathrm{~mm}$, increase of $\geq 0.5 \mathrm{~cm} /$ year or progression to an $\mathrm{AD}$ is an indication for surgery (18).

In a recent review of the literature of 925 patients with type B IMH, the 30-day and 3-year mortality, regardless of management, was $3.9 \%$ and as high as $14.3 \%$, respectively (10). After 1 year, $>50 \%$ of medically managed cases were either stable $(10 \%)$, had regressed $(30 \%)$ or had resolved $(60 \%)$. Of those patients who progressed, approximately $33 \%$ developed either a classic AD or aneurysm and $25 \%$ developed a localized AD or ULP. Less than $5 \%$ were complicated by rupture. The 30 -day and long-term mortality (3-5 years) for medical, open surgical and TEVAR ranged from $3 \%$ to $19 \%, 11 \%$ to $33 \%$, and $0 \%$ to $6 \%(3,10)$. Although the use of TEVAR to treat type B 
$\mathrm{IMH}$ is relatively recent, its preference over open surgical replacement is increasing (4).

An IMH may progress, regress, or even resolve over the first 30 days to 1 year, or as long as 5 years after presentation (10). Progression and adverse events are found in $3-14 \%$ of type B IMH. The large majority of aortic-related deaths occur within the first 8 days (i.e., the acute phase). Complications of IMH include progression of the disease, conversion to a classic $\mathrm{AD}$, aneurysm or pseudoaneurysm formation, branch vessel compromise and aortic rupture. Further studies are warranted to prove the safety and efficacy of pre-emptive TEVAR in uncomplicated type B IMHs and in patients with high-risk criteria. Treatment of acute patients deserves further consideration because of fragile tissues and risk of retrograde AD (12). Regardless of therapy, a life-long follow-up of these patients is justified.

\section{Acknowledgments}

None.

\section{Footnote}

Conflicts of Interest: The author has no conflicts of interest to declare.

\section{References}

1. Maslow A, Atalay MK, Sodha N. Intramural Hematoma. J Cardiothorac Vasc Anesth 2018;32:1341-62.

2. Alomari IB, Hamirani YS, Madera G, et al. Aortic intramural hematoma and its complications. Circulation 2014;129:711-6.

3. Mussa FF, Horton JD, Moridzadeh R, et al. Acute Aortic Dissection and Intramural Hematoma: A Systematic Review. JAMA 2016;316:754-63.

4. Harris KM, Braverman AC, Eagle KA, et al. Acute aortic intramural hematoma: an analysis from the International Registry of Acute Aortic Dissection. Circulation 2012;126:S91-6.

5. Riambau V, Bockler D, Brunkwall J, et al. Editor's Choice - Management of Descending Thoracic Aorta Diseases: Clinical Practice Guidelines of the European Society for Vascular Surgery (ESVS). Eur J Vasc Endovasc Surg 2017;53:4-52.

6. Paolucci M, Van Damme H, Boesmans E, et al. Type A intramural hematoma of aorta: An undervalued clinical entity. J Med Vasc 2018;43:206-12.

7. Krukenberg E. Beiträge zur Frage des Aneurysma dissecans. Fischer: Aus dem pathologischen Institut der Univ Leipzig 1919.

8. Moral S, Cuellar H, Avegliano G, et al. Clinical Implications of Focal Intimal Disruption in Patients With Type B Intramural Hematoma. J Am Coll Cardiol 2017;69:28-39.

9. Wu MT, Wang YC, Huang YL, et al. Intramural blood pools accompanying aortic intramural hematoma: $\mathrm{CT}$ appearance and natural course. Radiology 2011;258:705-13.

10. Evangelista A, Czerny M, Nienaber C, et al. Interdisciplinary expert consensus on management of type $\mathrm{B}$ intramural haematoma and penetrating aortic ulcer. Eur J Cardiothorac Surg 2015;47:209-17.

11. Uchida K, Imoto K, Takahashi M, et al. Pathologic characteristics and surgical indications of superacute type A intramural hematoma. Ann Thorac Surg 2005;79:1518-21.

12. Oderich GS, Karkkainen JM, Reed NR, et al. Penetrating Aortic Ulcer and Intramural Hematoma. Cardiovasc Intervent Radiol 2019;42:321-34.

13. Evangelista A, Dominguez R, Sebastia C, et al. Long-term follow-up of aortic intramural hematoma: predictors of outcome. Circulation 2003;108:583-9.

14. Hartlage GR, Palios J, Barron BJ, et al. Multimodality imaging of aortitis. JACC Cardiovasc Imaging 2014;7:60519.

15. Ciccone MM, Dentamaro I, Masi F, et al. Advances in the diagnosis of acute aortic syndromes: Role of imaging techniques. Vasc Med 2016;21:239-50.

16. Tanaka A, Leake S, Estrera AL. Management strategies in acute type B aortic intramural hematoma. Curr Opin Cardiol 2017;32:687-91.

17. Li L, Jiao Y, Zou J, et al. Thoracic Endovascular Aortic Repair versus Best Medical Treatment for High-Risk Type B Intramural Hematoma: A Systematic Review of Clinical Studies. Ann Vasc Surg 2018;52:273-9.

18. Sebastia C, Evangelista A, Quiroga S, et al. Predictive value of small ulcers in the evolution of acute type $\mathrm{B}$ intramural hematoma. Eur J Radiol 2012;81:1569-74.

Cite this article as: Haensig M. Type B intramural hematoma: focus on reasons for development and overlapping clinical disease. Ann Cardiothorac Surg 2019;8(4):494-496. doi: 10.21037/acs.2019.06.04 